\title{
Observations of galaxies at $z>6$. The properties of large, spectroscopic samples
}

\author{
Esther M. Hu, Lennox L. Cowie, and Yuko Kakazu \\ Institute for Astronomy, Honolulu, USA \\ email: hu@ifa.hawaii.edu
}

\begin{abstract}
Observed properties of spectroscopically confirmed galaxies at $z \gg 5$ and $z \gg 6$ based on selection from deep, multi-wavelength wide-field samples provide a picture of the current status of the properties of high-redshift galaxies and their evolution to yet higher redshifts.

In the current presentation, we use results of deep, wide-field spectroscopy with the multiobject Deimos spectrograph on Keck in combination with deep, wide-field multi-color imaging studies using the SUPRImeCAm CCD camera of Subaru for a number of fields, to evaluate the luminosity function of high-redshift galaxies and its evolution at $z>6$. High-redshift candidates are selected using both narrow-band Lyman alpha emission and broad-band colors with a high success-rate from a number of SuprimeCAm (0.5 degree FOV) fields.

Luminosity functions and Lyman $\alpha$ emission line profiles and equivalent widths appear similar between samples at $z \simeq 5.7$ and $z \simeq 6.5$, and the galaxy distribution is structured both spatially and in redshift. A large amount of cosmic variance is seen in the distribution of $z \gg 6$ galaxies from field to field.

The observed properties are discussed in relationship to their impact on strategies for complementary optical surveys of high-redshift galaxies, and in relationship to surveys at very different wavelengths (X-ray, far-infrared, and submillimeter) that cover the same regions.
\end{abstract}

Keywords. galaxies:high-redshift, infrared: galaxies, early Universe 